\title{
Heteroaromatic annulation studies on 2-[bis(methylthio)methylene]- 1-methyl-3-oxoindole: synthesis of novel heterocyclo[b] fused indoles
}

\author{
U. K. Syam Kumar, ${ }^{a}$ A. K. Yadav, ${ }^{a}$ C. Venkatesh, ${ }^{a}$ H. Ila*, and H. Junjappab \\ a, *Department of Chemistry, Indian Institute of Technology, Kanpur-208016, India \\ ${ }^{b}$ Bio Organics and Applied Materials Pvt. Ltd., \# B-64/1, Peenya Industrial Area, Peenya, \\ Bangalore-560058, India \\ E-mail: hila@iitk.ac.in
}

Dedicated to Professor P. T. Narasimhan on his $75^{\text {th }}$ birthday

(received 18 Mar 04; accepted 19 May 04; published on the web 22 May 04)

\begin{abstract}
Heteroannulation of 2-[Bis(methylthio)methylene]-1-methyl-3-oxoindole with $\beta$ substituted $\beta$-lithioaminoacrylonitrile, malononitrile and guanidine has been reported to yield novel substituted pyrido[3,2- $b]$ indoles and pyrimido[5,4- $b]$ indole derivatives in varying yields.
\end{abstract}

Keywords: Oxoindole, heterocyclization, $\delta$-carbolines, pyrimido[5,4- $b]$ indoles

\section{Introduction}

In the earlier work from this laboratory, we have described for the first time, the synthesis of 2[bis(methylthio)methylene]-1H-3-oxoindole $\mathbf{4}$ and the corresponding $N$-methyl analog $\mathbf{5}$ by careful in situ trapping of the unstable dipotassium salt $\mathbf{2}$ of the indoxyl enolate (obtained by base induced intramolecular condensation of 1) with carbon disulphide in an inert atmosphere and subsequent in situ methylation of the dithiolate salt $\mathbf{3}$ with either two or three equivalents of methyl iodide (Scheme 1). ${ }^{1}$ We have further carried out aromatic and heteroaromatic annulation studies on the $N$-methyl analog 5 with various allyl Grignard reagents and the anions derived from aryl/heteroaryl acetonitriles and antipyrine yielding novel benzo[c]-, naphtho[1,2-c]- and heterocyclo- fused carbazoles in good yields. ${ }^{1}$ Similarly, the heterocyclization studies on 5 with various 1,3-heterobinucleophilic species such as lithioacetonitrile, lithioaminocrotononitrile and 2- picolyllithium afforded the novel heterocyclo[b]- fused indoles such as pyrido[3,4- $b]$ - or pyrido[3,2-b]indoles and the corresponding indolo[3,2- $b]$ - quinolizinium salts in good yields. ${ }^{1}$ In continuation of these studies and in view of the biological importance of indolo[b]- fused heterocycles, ${ }^{2}$ we have now further investigated heterocyclization of ketene dithioacetal 5 with other bifunctional heterobinucleophiles such as $\beta$-substituted- $\beta$-lithioaminoacrylonitriles, 
malononitriles and guanidine yielding novel pyrido- and pyrimido fused indolo[b]- heterocycles. The results of these studies have been reported in the present paper.
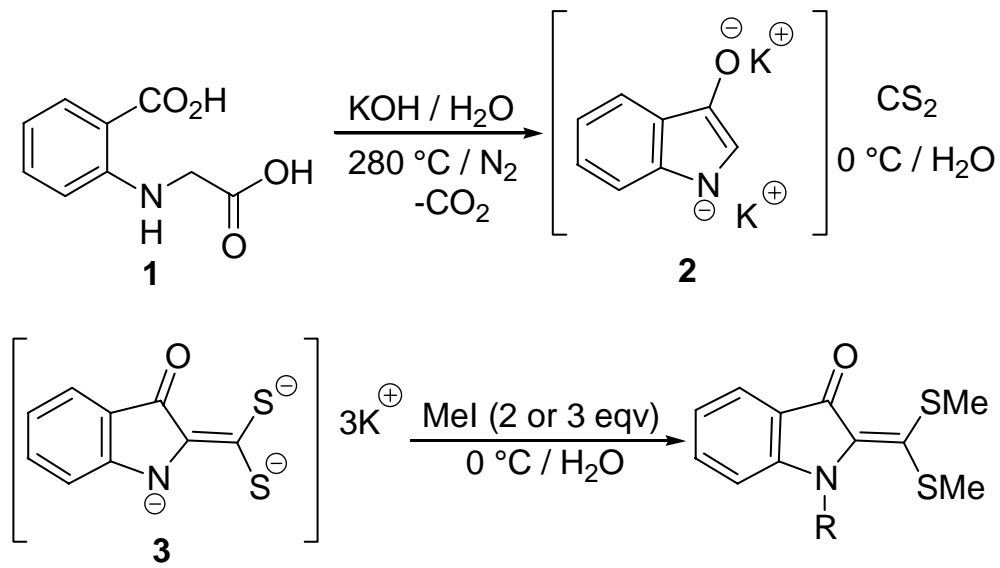

\section{Scheme 1}

$$
\begin{aligned}
& 4 \mathrm{R}=\mathrm{H} \\
& 5 \mathrm{R}=\mathrm{Me}
\end{aligned}
$$

\section{Results and Discussion}

We have earlier reported the reaction of $\beta$-lithioaminocrotononitrile generated in situ by treatment of one equivalent of $n$-butyllithium with two equivalents of acetonitrile at $-78{ }^{\circ} \mathrm{C}$ with 5 affording the corresponding 3-cyano-2,5-dimethyl-4-(methylthio)pyrido[3,2-b]indole ( $\delta$ carboline) in good yield. ${ }^{1,3}$ To further explore the scope of this reaction, we extended this method for the synthesis of various 2-substituted $\delta$-carbolines by addition of the corresponding $\beta$ substituted $\beta$-lithioaminoacrylonitriles 8 generated in situ by addition of lithioacetonitrile to various aromatic and aliphatic nitriles as shown in Scheme 2. ${ }^{3}$

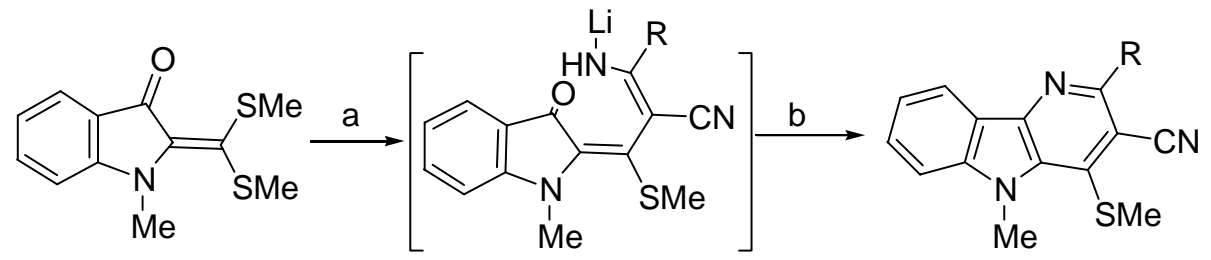

5

7

a) $\mathrm{MeCN}$ (1 eqv) / RCN (1 eqv) / $n$-BuLi (1 eqv) / $-78^{\circ} \mathrm{C}$ b) $80^{\circ} \mathrm{C} / 28 \mathrm{~h}$

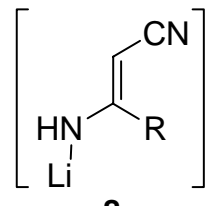

6-8a, $\mathrm{R}=\mathrm{Ph}, 62 \%$

8

b, $\mathrm{R}=4-\mathrm{MeOC}_{6} \mathrm{H}_{4}, 63 \%$

c, $\mathrm{R}=2$-thienyl, $58 \%$

d, $\mathrm{R}=\mathrm{MeOCH}_{2}, 64 \%$

\section{Scheme 2}


Thus the 2-substituted 3-cyano-4-(methylthio)pyrido[3,2-b]- indoles 7a-d were obtained in 58-64\% overall yields through initial 1,4-conjugate addition of lithioaminoacrylonitriles 8a-d followed by in situ heterocyclization of adducts 6a-d as observed earlier. The present method thus provides an efficient three component route for substituted $\delta$-carbolines. It should be noted that the reported methods for the synthesis of $\delta$-carbolines are scant in the literature in comparison to those for $\beta$ - or $\gamma$-carboline derivatives. ${ }^{2 \mathrm{~g}, 4}$ The structures and regiochemistry of all newly synthesized $\delta$ - carbolines 7a-d were confirmed with the help of spectral and analytical data.

In an another approach towards functionalized pyrido[3,2-b]indole frameworks, the ketene dithioacetal 5 was reacted with malononitrile in presence of sodium methoxide in refluxing methanol, when the reaction mixture after work-up yielded the corresponding 2-methoxy-3cyano-5-methyl-4-(methylthio)pyrido[3,2-b]indole 10 in 45\% yield (Scheme 3). This is again an interesting example of three component heterocyclization leading to a substituted pyrido[3,2$b$ ]indole through attack of methoxy group on one of the nitrile groups in the intermediate conjugate adduct 5 followed by cyclodehydration.

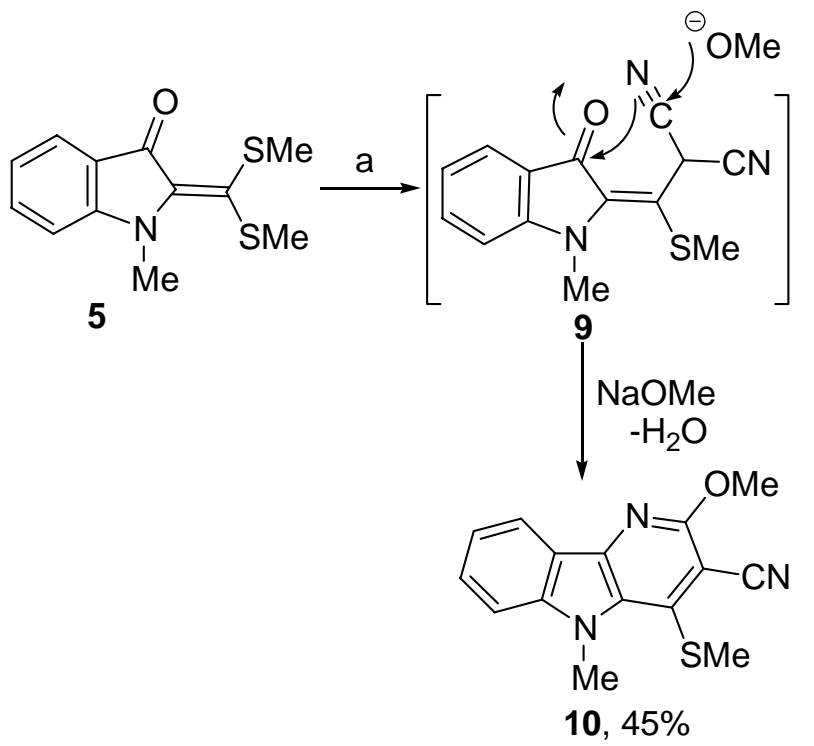

a) $\mathrm{CH}_{2}(\mathrm{CN})_{2} / \mathrm{NaOMe}, \mathrm{MeOH} / \Delta$.

\section{Scheme 3}

We next investigated the heterocyclization of $\mathbf{1}$ with guanidine nitrate ${ }^{5}$ with a view to synthesize the pyrimido[5,4-b]indole framework ${ }^{6}$ as shown in Scheme 4. Thus when 5 was reacted with guanidine nitrate in the presence of sodium isopropoxide in refluxing isopropanol, the expected 2-amino-4-(methylthio)-5-methylpyrimido[5,4- $b$ ]indole 11 was obtained in 52\% yield. On the other hand, when 5 was reacted with guanidine nitrate in the presence of sodium methoxide or sodium ethoxide in refluxing methanol (or ethanol), the corresponding 4-methoxyand 4-ethoxypyrimido[5,4- $b$ ] indoles 12a-b were obtained in 48\% and 50\% yields respectively. 
Apparently the ketene dithioacetal $\mathbf{5}$ undergoes thiomethyl displacement to give the corresponding $O, S$-acetals 13a-b which further reacted with guanidine nitrate to afford the observed fused pyrimidines 12a-b (Scheme 4). The sodium isopropoxide being a hindered base does not undergo displacement with $\mathbf{5}$ under these conditions.

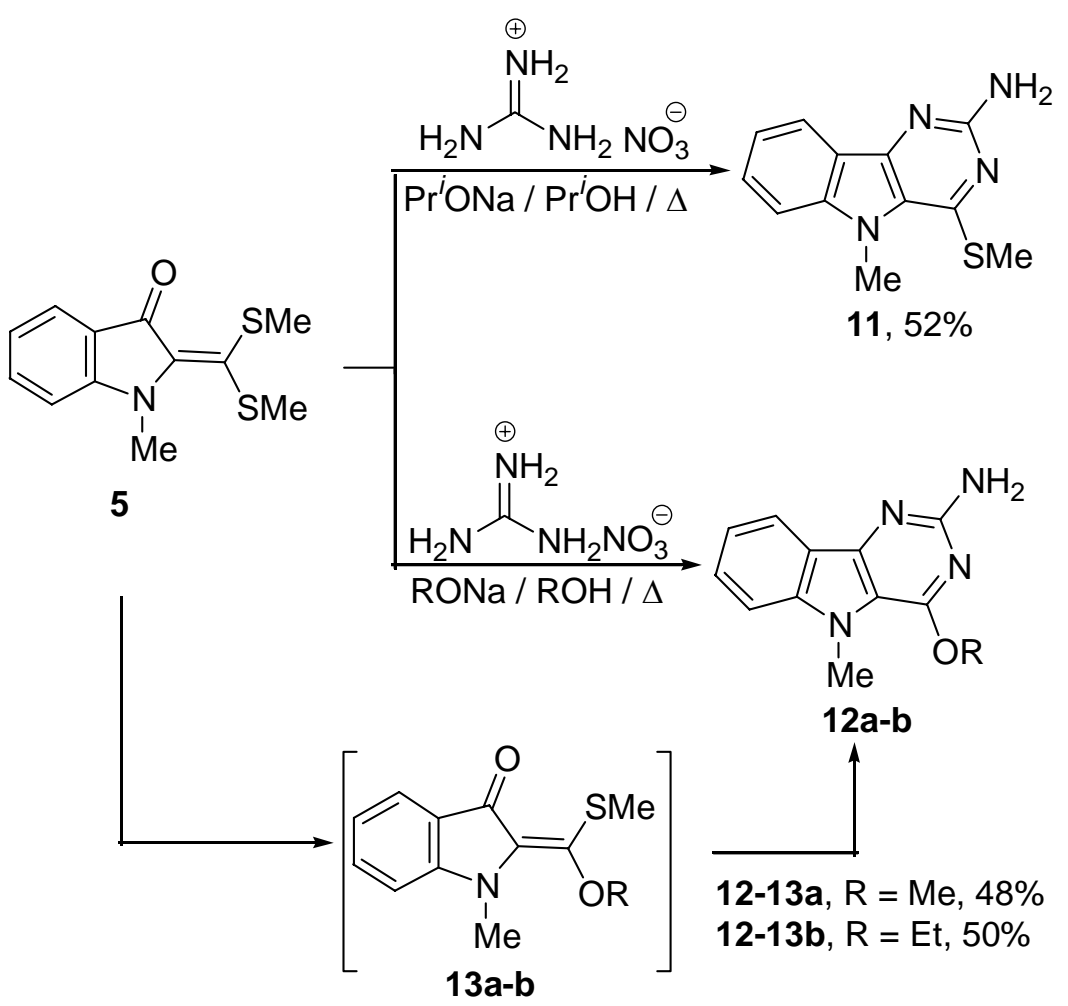

\section{Scheme 4}

In summary, heterocyclization studies on 2-[bis(methylthio)methylene]-2,3-dihydro-3-oxo-1methylindole 5 with $\beta$-substituted- $\beta$-lithioaminoacrylonitriles, malononitrile and guanidine yield novel substituted pyrido[3,2- $b$ ]indole and pyrimido[5,4- $b]$ indole derivatives in moderate to good yields.

\section{Experimental Section}

General Procedures. ${ }^{1} \mathrm{H}$ NMR (400 MHz) and ${ }^{13} \mathrm{C}$ NMR (100 MHz) spectra were recorded in $\mathrm{CDCl}_{3}$ and TMS was used as an internal reference. Melting points are uncorrected. Chromatographic purification was conducted by column chromatography using 60-120 mesh silica gel obtained from Acme Synthetic Chemicals. THF was distilled over sodium/benzophenone prior to use. 4-Methoxybenzonitrile, 2-cyanothiophene, methoxyacetonitrile, malononitrile, guanidine nitrate, $n$-BuLi(1.6M in hexane) were purchased 
from standard firms and used directly. The 2-bis(methylthio)methylene-1-methyl-3-oxoindole (5) was prepared according to the earlier reported procedure. ${ }^{1}$

General procedure for the generation of 2-substituted 2-lithioaminoacrylonitriles 8 and their reactions with 5: Synthesis of 2-substituted 3-cyano-4-(methylthio)pyrido[3,2b]indoles 7a-d

To stirred solution of acetonitrile $(0.4 \mathrm{~mL}, 7.6 \mathrm{mmol})$ in dry THF (25 mL), $n$-BuLi (7.5 mmol, $1.6 \mathrm{M}$ in hexane) was added under $\mathrm{N}_{2}$ at $-78{ }^{\circ} \mathrm{C}$ and the reaction mixture was stirred for $0.5 \mathrm{~h}$ at the same temperature. To the resulting white suspension of lithioacetonitrile, a solution of alkyl/aryl or heteroaryl nitrile $(7.5 \mathrm{mmol})$ in dry THF $(10 \mathrm{~mL})$ was added dropwise and the reaction mixture was further stirred at the same temperature for $0.5 \mathrm{~h}$ to give a light reddish solution of 2-substituted 2-lithioaminoacrylonitriles. To these in situ generated 2lithioaminoacrylonitriles, a solution of 5 (1.26 g, $5.0 \mathrm{mmol})$ in dry THF (25 mL) was added dropwise at $-78{ }^{\circ} \mathrm{C}$. The reaction mixture was brought to room temperature and refluxed with stirring for 40-48 h (monitored by TLC). It was then cooled, poured into saturated $\mathrm{NH}_{4} \mathrm{Cl}$ solution, extracted with $\mathrm{CHCl}_{3}(2 \times 100 \mathrm{~mL})$ and the combined organic layers were washed with water, dried $\left(\mathrm{Na}_{2} \mathrm{SO}_{4}\right)$ and concentrated to give crude $\mathbf{7 a - 7 d}$, which were purified by column chromatography over silica gel using hexane-EtOAc (19:1) as eluent.

3-Cyano-5- $\boldsymbol{N}$-methyl-4-(methylthio)-2-phenylpyrido[3,2-b]indole (7a). Colorless crystals (ether-hexane); mp 171-172 ${ }^{\circ} \mathrm{C}$ Yield 62\%; IR (KBr): 2210, 1546, $1460 \mathrm{~cm}^{-1}$; ${ }^{1} \mathrm{H}$ NMR $\left(400 \mathrm{MHz}, \mathrm{CDCl}_{3}\right): \delta 2.71$ (s, 3H, $\left.\mathrm{SCH}_{3}\right), 4.42$ (s, 3H, $\mathrm{NCH}_{3}$ ), 7.36-7.41 (m, 1H, ArH), 7.497.56 (m, 4H, ArH), 7.67-7.71 (m, 1H, ArH), 7.95-7.97 (m, 2H, ArH), 8.45 (d, $J=8.0 \mathrm{~Hz}, 1 \mathrm{H}$, ArH); MS: (m/z, \%): 329 ( $\left.\mathrm{M}^{+}, 100\right), 314$ (31); Anal. Calcd. for $\mathrm{C}_{20} \mathrm{H}_{15} \mathrm{~N}_{3} \mathrm{~S}$ (329.43): C, 72.92; $\mathrm{H}$, 4.59; N, 12.76\%. Found: C, 73.19; H, 4.95; N, 12.59\%.

3-Cyano-2-(4-methoxyphenyl)-5- $N$-methyl-4-(methylthio)pyrido[3,2-b]indole (7b). Colorless crystals (ether-hexane); mp 209-210 ${ }^{\circ} \mathrm{C}$ Yield 63\%; IR (KBr): 2200, 1596, $1460 \mathrm{~cm}^{-1}$; ${ }^{1} \mathrm{H}$ NMR $\left(400 \mathrm{MHz}, \mathrm{CDCl}_{3}\right): \delta 2.66$ (s, 3H, $\left.\mathrm{SCH}_{3}\right), 3.88$ (s, 3H, $\left.\mathrm{OCH}_{3}\right), 4.23$ (s, 3H, $\left.\mathrm{NCH}_{3}\right), 7.01$ (d, $J=$ $8.8 \mathrm{~Hz}, 2 \mathrm{H}, \mathrm{ArH}), 7.05-7.09$ (m, 1H, ArH), 7.31-7.33 (m, 2H, ArH), 7.77 (d, J = 8.0 Hz, 1H, $\mathrm{ArH}), 7.95$ (d, $J=8.8 \mathrm{~Hz}, 2 \mathrm{H}, \mathrm{ArH}) ;{ }^{13} \mathrm{C} \mathrm{NMR}$ (100 MHz, $\mathrm{CDCl}_{3}$ ): 13.02, 32.95, 55.47, 108.32, 109.54, 114.45, 115.21, 117.63, 118.66, 119.61, 125.70, 128.61, 128.62, 137.94, 146.57, 150.30, 151.71, 161.13, 162.09; MS: (m/z, \%): 359 ( $\left.\mathrm{M}^{+}, 100\right)$; Anal. Calcd. for $\mathrm{C}_{21} \mathrm{H}_{17} \mathrm{~N}_{3} \mathrm{OS}$ (359.45): C, 70.17; H, 4.77; N, 11.69\%. Found: C, 70.41; H, 4.68; N, 11.61\%.

3-Cyano-5- $N$-methyl-4-methylthio-2-(thienyl)pyrido[3,2-b]indole (7c). Colorless crystals (ether-hexane); mp 228-229 ${ }^{\circ} \mathrm{C}$ Yield 58\%; IR (KBr): 2202, 1550, $1490 \mathrm{~cm}^{-1}$; ${ }^{1} \mathrm{H}$ NMR $\left(400 \mathrm{MHz}, \mathrm{CDCl}_{3}\right): \delta 2.64\left(\mathrm{~s}, 3 \mathrm{H}, \mathrm{SCH}_{3}\right), 4.18$ (s, 3H, $\left.\mathrm{NCH}_{3}\right), 7.04-7.16(\mathrm{~m}, 2 \mathrm{H}, \mathrm{ArH}), 7.26-$ 7.34 (m, 2H, ArH), 7.50 (d, $J=5.12 \mathrm{~Hz}, 1 \mathrm{H}, \operatorname{ArH}), 7.68$ (d, $J=5.1 \mathrm{~Hz}, 1 \mathrm{H}, \operatorname{ArH}), 7.76(\mathrm{~d}, J=$ $7.8 \mathrm{~Hz}, 1 \mathrm{H}, \mathrm{ArH}) ;{ }^{13} \mathrm{C} \mathrm{NMR}\left(100 \mathrm{MHz}, \mathrm{CDCl}_{3}\right)$ : 13.02, 32.91, 106.86, 109.54, 112.10, 113.51, 117.23, 118.66, 119.66, 125.83, 127.19, 128.47, 129.82, 138.00, 138.52, 146.64, 160.64, 183.17; MS: (m/z, \%): $335\left(\mathrm{M}^{+}, 100\right)$; Anal. Calcd. for $\mathrm{C}_{18} \mathrm{H}_{13} \mathrm{~N}_{3} \mathrm{~S}_{2}$ (335.45): C, 65.45; H, 3.91; N, 12.53\%. Found: C, 65.69; H, 4.02; N, 12.41\%. 
3-Cyano-2-methoxymethyl-5- $N$-methyl-4-(methylthio)pyrido[3,2-b]indole (7d). Colorless crystals (ether-hexane); mp 180-181 ${ }^{\circ} \mathrm{C}$; Yield 64\%; IR (KBr): 2202, 1600, $1541 \mathrm{~cm}^{-1}$; ${ }^{1} \mathrm{H}$ NMR $\left(400 \mathrm{MHz}, \mathrm{CDCl}_{3}\right): \delta 2.67\left(\mathrm{~s}, 3 \mathrm{H}, \mathrm{SCH}_{3}\right), 3.62$ (s, 3H, $\left.\mathrm{OCH}_{3}\right), 4.37$ (s, 3H, $\left.\mathrm{NCH}_{3}\right), 4.91(\mathrm{~s}, 2 \mathrm{H}$, $\mathrm{CH}_{2} \mathrm{OCH}_{3}$ ), 7.38 (t, $\left.J=7.9 \mathrm{~Hz}, 1 \mathrm{H}, \mathrm{ArH}\right), 7.50$ (d, $\left.J=8.2 \mathrm{~Hz}, 1 \mathrm{H}, \mathrm{ArH}\right), 7.65-7.69$ (m, 1H, $\mathrm{ArH}), 8.42$ (d, $J=7.8 \mathrm{~Hz}, 1 \mathrm{H}, \mathrm{ArH}) ;{ }^{13} \mathrm{C} \mathrm{NMR}$ (100 MHz, $\mathrm{CDCl}_{3}$ ): 20.64, 32.21, 59.20, 74.68, 109.50, 111.80, 116.60, 121.08, 121.22, 122.24, 130.13, 130.91, 132.76, 144.00, 144.45, 152.29; MS: (m/z, \%): $297\left(\mathrm{M}^{+}, 24\right), 267$ (100); Anal. Calcd. for $\mathrm{C}_{16} \mathrm{H}_{15} \mathrm{~N}_{3} \mathrm{OS}$ (297.38): C, 64.62; $\mathrm{H}$, 5.08; N, 14.13\%. Found: C, 64.79; H, 5.22; N, 14.31\%.

\section{Procedure for cycloannulation of 5 with malononitrile}

A solution of 5 (1.26 g, $5 \mathrm{mmol}$ ) in dry methanol was added to a solution of malononitrile (0.61 g, $5 \mathrm{mmol}$ ) and sodium methoxide (from 0.05 mole of sodium) in $50 \mathrm{~mL}$ of dry methanol and the reaction mixture was refluxed for $12 \mathrm{~h}$. The solvent was removed under reduced pressure, the residue was diluted with cold water and extracted with $\mathrm{CHCl}_{3}(2 \times 50 \mathrm{~mL})$. The $\mathrm{CHCl}_{3}$ layer was dried $\left(\mathrm{Na}_{2} \mathrm{SO}_{4}\right)$ and concentrated to give crude product which was then purified by column chromatography over silica gel using hexane-EtOAc (6: 1) as eluent.

3-Cyano-2-methoxy-5- $N$-methyl-4-(methylthio)pyrido[3,2-b]indole (10). Colorless crystals (ether-hexane); mp 228-229 ${ }^{\circ} \mathrm{C}$ Yield 45\%; IR (KBr): 2202, 1550, $1490 \mathrm{~cm}^{-1}$; ${ }^{1} \mathrm{H}$ NMR $\left(400 \mathrm{MHz}, \mathrm{CDCl}_{3}\right): \delta 2.17$ (s, 3H, $\left.\mathrm{SCH}_{3}\right), 3.87\left(\mathrm{~s}, 3 \mathrm{H}, \mathrm{OCH}_{3}\right), 3.97\left(\mathrm{~s}, 3 \mathrm{H}, \mathrm{NCH}_{3}\right), 7.06(\mathrm{t}, \mathrm{J}=$ $7.08 \mathrm{~Hz}, 1 \mathrm{H}, \mathrm{ArH}), 7.24$ (d, J = 8.32 Hz, 1H, ArH), 7.35-7.39 (m, 1H, ArH), 7.74 (d, $J=8.0 \mathrm{~Hz}$, $1 \mathrm{H}, \mathrm{ArH}$ ); Anal. Calcd. for $\mathrm{C}_{15} \mathrm{H}_{13} \mathrm{~N}_{3} \mathrm{SO}$ (283.45): C, 63.58; H, 4.62; N, 14.83\%. Found: C, 63.69; H, 4.52; N, 14.74\%.

\section{Reaction of 5 with guanidine nitrate}

\section{(a) in the presence of sodium isopropoxide}

A solution of 5 (1.26 g, $5.0 \mathrm{mmol})$ in dry isopropanol was added to a suspension of guanidine nitrate (0.61 g, $5.0 \mathrm{mmol})$ and sodium isopropoxide (0.05 mmol of sodium) in dry isopropanol $(50 \mathrm{~mL})$ and the reaction was refluxed for 12-14 h. The solvent was removed under reduced pressure, the residue diluted with cold water and extracted with $\mathrm{CHCl}_{3}(2 \times 50 \mathrm{~mL})$. The $\mathrm{CHCl}_{3}$ layer was dried $\left(\mathrm{Na}_{2} \mathrm{SO}_{4}\right)$ and concentrated to give crude product which was then purified by column chromatography over silica gel using hexane-EtOAc (6: 1) as the eluent.

2-Amino-5- $\boldsymbol{N}$-methyl-4-(methylthio)pyrimido[5,4-b]indole (11). Light yellow crystals (methanol); mp 227-228 ${ }^{\circ} \mathrm{C}$ Yield 52\%; IR (KBr): 3250, 3100, 1650, 1600, $1500 \mathrm{~cm}^{-1}$; ${ }^{1} \mathrm{H}$ NMR (400 MHz, $\mathrm{CDCl}_{3}$ ): $\delta 3.01$ (s, 3H, $\mathrm{SCH}_{3}$ ), 4.12 (s, 3H, $\mathrm{NCH}_{3}$ ), 7.10-7.26 (m, 1H, ArH), 7.357.37 (m, 1H, ArH), 7.55-7.59 (m, 1H, ArH), 8.20 (d, $J=7.9$ Hz, 1H, ArH); MS: (m/z, \%): 244 $\left(\mathrm{M}^{+}, 100\right)$; Anal. Calcd. for $\mathrm{C}_{12} \mathrm{H}_{12} \mathrm{~N}_{4} \mathrm{~S}$ (244.32): C, 58.99; H, 4.95; N, 22.93\%. Found: C, 58.79; H, 4.62; N, 22.81\%.

(b) in methanol or ethanol

A solution of 5 (1.26 g, $5.0 \mathrm{mmol})$ in dry methanol/ethanol was added to a suspension of guanidine nitrate $(0.61 \mathrm{~g}, 5.0 \mathrm{mmol})$ and sodium methoxide or ethoxide (from $0.05 \mathrm{mmol}$ of 
sodium) in $50 \mathrm{~mL}$ of the respective alcohol and the reaction mixture was refluxed for 12-14 h. The solvent was removed under reduced pressure, the residue was diluted with cold water and extracted with $\mathrm{CHCl}_{3}(2 \times 50 \mathrm{~mL})$. The organic layer was dried $\left(\mathrm{Na}_{2} \mathrm{SO}_{4}\right)$ and concentrated to give crude product which was then purified by column chromatography over silica gel using hexane-EtOAc (6:1) as eluent.

2-Amino-5- $N$-methyl-4-(methoxy)pyrimido[5,4-b]indole (12a). Colorless crystals (Etherhexane); mp 145-146 ${ }^{\circ} \mathrm{C}$ Yield 48\%; IR (KBr): 3466, 3295, 3161, 3100, 1650, 1600, $1500 \mathrm{~cm}^{-1}$; ${ }^{1} \mathrm{H}$ NMR (400 MHz, $\mathrm{CDCl}_{3}$ ): $\delta 2.86$ (s, 3H, $\mathrm{SCH}_{3}$ ), 3.99 (s, 3H, $\mathrm{NCH}_{3}$ ), 4.92 (brs, $2 \mathrm{H}, \mathrm{NH}_{2}$ ), 7.17-7.21 (m, 1H, ArH), 7.33-7.35 (m, 1H, ArH), 7.50-7.53 (m, 1H, ArH), 8.14 (d, J= 8.0 Hz, $1 \mathrm{H}, \mathrm{ArH}) ;{ }^{13} \mathrm{C}$ NMR (100 MHz, $\left.\mathrm{CDCl}_{3}\right): 31.64,53.49,109.45,115.12,119.18,119.94,121.26$, 128.34, 141.85, 146.40, 156.82, 157.68; MS: (m/z, \%): 228 ( $\left.\mathrm{M}^{+}, 100\right), 213$ (56); Anal. Calcd. for $\mathrm{C}_{12} \mathrm{H}_{12} \mathrm{~N}_{4} \mathrm{~S}$ (228.25): C, 63.14; H, 5.30; N, 24.55\%. Found: C, 63.39; H, 5.45; N, 24.32\%.

2-Amino-5- $N$-methyl-4-(ethoxy)pyrimido[5,4-b]indole (12b). White crystals (ether-hexane); mp 225-226 ${ }^{\circ} \mathrm{C}$ Yield 50\%; IR (KBr): 3479, 3287, 3151, 1650, 1600, $1500 \mathrm{~cm}^{-1}$; ${ }^{1} \mathrm{H}$ NMR (400 MHz, $\left.\mathrm{CDCl}_{3}\right): \delta 1.48\left(\mathrm{t}, J=7.0 \mathrm{~Hz}, 3 \mathrm{H}, \mathrm{CH}_{3}\right), 4.00\left(\mathrm{~s}, 3 \mathrm{H}, \mathrm{NCH}_{3}\right) 4.56$ (q, $J=7.08,2 \mathrm{H}$, $\mathrm{CH}_{2} \mathrm{CH}_{3}$ ), 4.83 (brs, $2 \mathrm{H}, \mathrm{NH}_{2}$ ), 7.17-7.21 (m, 1H, ArH), 7.35 (d, $\left.J=8.6 \mathrm{~Hz}, 1 \mathrm{H}, \mathrm{ArH}\right), 7.49-7.53$ (m, 1H, ArH), 8.14 (d, $J=7.8 \mathrm{~Hz}, 1 \mathrm{H}, \mathrm{ArH}) ;{ }^{13} \mathrm{C} \mathrm{NMR}\left(100 \mathrm{MHz}, \mathrm{CDCl}_{3}\right): 14.57,31.58,62.18$, 109.43, 115.23, 119.43, 120.04, 121.22, 128.26, 141.90, 146.21, 156.89, 157.43; MS: (m/z, \%): 242 ( $\left.\mathrm{M}^{+}, 100\right), 227$ (50); Anal. Calcd. for $\mathrm{C}_{13} \mathrm{H}_{14} \mathrm{~N}_{4} \mathrm{O}$ (242.28): C, 64.45; H, 5.82; N, 23.13\%. Found: C, 64.65; H, 5.60; N, 23.41\%.

\section{Acknowledgements}

Financial assistance by DST, New Delhi is acknowledged.

\section{References}

1. Basaveswara Rao, M.V.; Syam Kumar, U. K.; Ila, H.; Junjappa, H. Tetrahedron 1999, 55, 11563.

2. (a) Abouabdellah, A.; Dodd, R. H. Tetrahedron Lett. 1998, 39, 2119. (b) Molina, P.; Fresnada, P. M.; Sanz, N. A.; Foces-Foces, C.; Ramirez de Arellano, M. C. Tetrahedron 1998, 54, 9623. (c) Papamicael, C.; Dupas, G.; Queguiner, G.; Bourguignon, M. Heterocycles 1998, 47, 991. (d) Dubois, C.; Dorey, G.; Potier, P.; Dodd, R.A. Tetrahedron Asymmetry 1995, 6, 455. (e) Cimanga, K.; Bruyne, T. D.; Pieters, L.; Claeys, M.; Vietinck, K. A. Tetrahedron Lett. 1996, 37, 1703. (f) Bolton, D.; Forbes, I. T.; Hayward, C. J.; Piper, D. C.; Thomas, D. R.; Thompson, M.; Upton, N. Biorg. Med. Chem. Lett. 1993, 3, 1941. (g) Abramovitch, R.A. Can. J. Chem. 1960, 38, 2273. (h) Kawasaki, T.; Terashima, R.; Sakaguchi, K. -e.; Sekiguchi, H.; Sakamoto, M. Tetrahedron Lett. 1996, 37, 7527. 
3. Gupta, A. K.; Ila, H.; Junjappa, H. Tetrahedron 1990, 46, 3703.

4. (a) Yang, C. -C.; Sun, P.-J.; Fang, J. M. J. Chem. Soc., Chem. Commun. 1994, 2629. (b) Merour, J. Y.; Merour, A. Synthesis 1994, 767. (c) Aezel, I. E.; Rocca, P.; Marsais, F.; Gadard, A.; Queguiner, G. J. Heterocycl. Chem. 1997, 34, 1205. (d) Abramovitch, R. A.; Adams, K.; Notation, A.D. Can. J. Chem. 1960, 38, 2723.

5. (a) Chauhan, S. M. S.; Junjappa, H Synthesis 1974, 880. (b) Chauhan, S. M. S.; Junjappa, H. Tetrahedron 1976, 32, 1779.

6. (a) Suvorov, N. N.; Chernov, V. A.; Velezheva, V. S.; Ershova, Yu. A.; Simakov, S. V.; Sevodin, V. P. Khim.-Farm. Zh. 1981, 15, 27; Chem. Abstr. 1982, 96, 85492s. (b) Velezheva, V. S.; Simakov, S. V.; Dymov, V. N.; Suvorov, N. N. Khim. Getero. Soed. 1980, 6, 851; Chem. Abstr. 1981, 94, 24878e. 